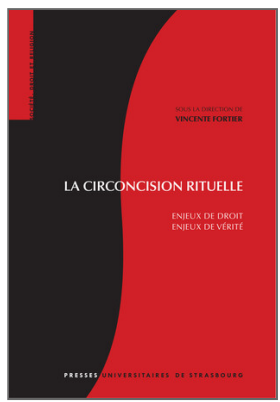

Vincente Fortier (dir.)

La circoncision rituelle

Enjeux de droit, enjeux de vérité

Presses universitaires de Strasbourg

\title{
La circoncision rituelle au regard du droit français
}

Vincente Fortier, Juliette Dugne, Juliette Lelieur et François Vialla

DOI : $10.4000 /$ books.pus. 14445

Éditeur : Presses universitaires de Strasbourg

Lieu d'édition : Strasbourg

Année d'édition : 2016

Date de mise en ligne : 28 mai 2019

Collection : Société, droit et religion

EAN électronique : 9791034404179

\section{Cbooks}

http://books.openedition.org

Édition imprimée

Date de publication : 7 juin 2016

Ce document vous est offert par Université de Strasbourg

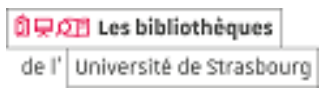

Référence électronique

FORTIER, Vincente; i et al. La circoncision rituelle au regard du droit français In : La circoncision rituelle

Enjeux de droit, enjeux de vérité [en ligne]. Strasbourg : Presses universitaires de Strasbourg, 2016

(généré le 10 octobre 2021). Disponible sur Internet : <http://books.openedition.org/pus/14445>.

ISBN : 9791034404179 . DOI : https://doi.org/10.4000/books.pus.14445. 


\title{
La circoncision rituelle au regard du droit français
}

\author{
VINCENTE ForTier \\ Juliette Dugne \\ Juliette Lelieur \\ FranÇOIS VIALLA
}

\begin{abstract}
a circoncision rituelle est une énigme pour le juriste français en quête de _certitude et qui peut difficilement se satisfaire d'un raisonnement fondé sur la tolérance sociale pour asseoir la légitimité et la validité d'un rite qui, en tout état de cause, consomme une atteinte à l'intégrité physique du corps de l'enfant. Écartant par conséquent du champ de la réflexion, le socle mou de la tolérance, pour circonscrire le débat au seul domaine juridique, incluant, bien évidemment, la prise en compte de la liberté religieuse, la problématique de la circoncision rituelle mobilise différentes ressources juridiques à l'aune desquelles elle doit être interrogée. Le questionnement est multiple.

En effet et d'une part, la circoncision entre-t-elle en conflit avec le principe d'inviolabilité du corps humain tel qu'il est désormais posé expressément par le Code civil ? La voie ouverte par le droit de la santé, dans ses développements doctrinaux les plus récents, permet-elle de valider cette pratique et, le cas échéant, sous quelles conditions?

D'autre part, le droit de la famille, convié au débat et interprété par les juges judiciaires, fournit-il une réponse et précisément un cadre d'exécution de cette pratique offrant aux parents et à l'enfant une protection suffisante, même si demeure le caractère contra legem de la pratique ? Et, dans l'hypothèse où les conditions posées par le juge civil ne sont pas respectées, quelles
\end{abstract}


sont les sanctions possibles à l'encontre du parent ayant méconnu les exigences prétoriennes ? En outre, lorsqu'un dommage advient, quels sont les recours possibles et les modalités de la réparation, des points de vue civil et/ou administratif ?

Enfin, et bien que la circoncision rituelle en tant que telle bénéficie de facto d'une totale impunité au regard du droit pénal, le nouvel article 227-24-1 du Code pénal visant de manière générale les mutilations sexuelles ne pourrait-il pas conduire à une remise en cause de la pratique ?

La situation de la circoncision rituelle est complexe. Elle appelle un examen différencié selon le corpus juridique sollicité. C'est donc selon quatre axes correspondant aux différentes strates du questionnement que la réflexion s'organise : l'inviolabilité du corps humain ; la religion de l'enfant, prérogative parentale ; la réparation des conséquences dommageables de l'acte de circoncision ; la remise en cause indirecte de l'impunité pénale.

\section{L'INVIOLABILITÉ DU CORPS HUMAIN}

Comme l'écrit Gérard Cornu dans son ouvrage de droit civil ${ }^{1}$, "Rempli d'une force coutumière immémoriale, le principe de l'inviolabilité du corps humain est de la plus haute tradition ": Noli me tangere fondamental "comme bouclier de liberté corporelle $»^{2}$ ou encore qualifié par André Demichel comme plus vieux principe normatif qui soit en la matière ${ }^{3}$.

Jusqu'en 1994, le droit des personnes tout en prenant en considération le corps humain, le désignait toutefois rarement distinctement de la personne. La protection du corps était (et est toujours) assurée par le Code civil (article 1382 "Tout fait quelconque de l'homme qui cause à autrui un dommage, oblige celui par la faute duquel il est arrivé, à le réparer ») et par le Code pénal à travers l'incrimination de tous les délits contre la personne.

Il a fallu attendre 1994 pour que le droit se préoccupe du corps en tant que tel, afin d'en assurer la protection. La loi civile, désormais, consacre le principe traditionnel et " proclame solennellement la valeur qu'elle reconnaît au corps humain $"^{4}$.

1 Cornu Gérard, Droit civil. Introduction, Les personnes, les biens, Paris, Montchrestien, Précis Domat/Droit privé, $7^{\mathrm{e}}$ édition, 1995, p. 170.

2 Carbonnier Jean, cité par Mémeteau Gérard, Cours de droit médical, Bordeaux, Les Études hospitalières, $4^{\mathrm{e}}$ édition, 2010, p. 379.

3 Demichel André, "Médecine et droit : bilan provisoire d'une cohabitation problématique ", in Etudes offertes à Jean-Marie Auby, Paris, Dalloz, 1992, p. 720.

4 Connu Gérard, op. cit., p. 171. 
L'article 16-1 du Code civil dispose, en effet :

"Chacun a droit au respect de son corps.

Le corps humain est inviolable.

Le corps humain, ses éléments et ses produits ne peuvent faire l'objet d'un droit patrimonial. "

Les principes ainsi énoncés sont généraux : ils protègent toute personne contre toute atteinte à son corps. Ils sont également d'ordre public, s'accompagnant d'un panel de sanctions et dont le respect est assuré par le juge judiciaire, "érigé en gardien du corps humain ". C'est ainsi qu'aux termes de l'article 16-2duCodecivil, « Lejugepeutprescriretoutes mesurespropresà empêcher oufairecesseruneatteinteilliciteau corps humainou desagissementsillicites portant sur des éléments ou des produits de celui-ci ».

Le principe d'inviolabilité du corps humain («ou de la personne en son corps ») posé à l'article 16-1 alinéa 2 du Code civil comprend la notion d'intégrité visée par l'article 16-3 du même Code. En effet, le principe fixé à l'article 16-1, selon lequel nul ne peut être contraint de subir une atteinte à son corps, doit être interprété en articulation avec l'article 16-3 qui dispose :

« II ne peut être porté atteinte à l'intégrité du corps humain qu'en cas de nécessité médicale pour la personne ou à titre exceptionnel dans l'intérêt thérapeutique d'autrui.

Le consentement de l'intéressé doit être recueilli préalablement hors le cas où son état rend nécessaire une intervention thérapeutique à laquelle il n'est pas à même de consentir ".

Le principe de l'intégrité est écarté lorsque deux conditions se trouvent réunies : d'une part, une nécessité médicale (antérieurement nécessité thérapeutique) et d'autre part, le consentement de l'intéressé sous réserve de ne pas contrevenir à l'ordre public. La protection du corps contre toute atteinte non justifiée par une nécessité médicale s’applique aux majeurs comme aux mineurs.

En subordonnant l'intervention sur le corps à l'existence d'une nécessité médicale et en disposant que " le consentement de l'intéressé doit être recueilli préalablement ", l'article 16-3 alinéa 2 du Code civil réitère les conditions posées dans le Code de la santé publique (article 4127-41) et dans le Code de déontologie médicale (article 41) : "Aucune intervention mutilante ne peut être pratiquée sans motif médical sérieux et, sauf urgence ou impossibilité, sans information de l'intéressé et sans son consentement ».

Le principe d'intangibilité du corps humain apparaît primordial mais est-il absolu ? La circoncision rituelle représente à cet égard une « exception à l'intangibilité du corps humain puisqu'elle consomme une atteinte irréversible à 
l'intégrité corporelle d'un garçon et qu'elle intervient en toute hypothèse sans le consentement de celui-ci ${ }^{6}$.

Examinée sous l'angle de l'inviolabilité du corps humain et du respect de son intégrité, la circoncision rituelle, qui consiste en une ablation totale ou partielle du prépuce de l'enfant, réalise une atteinte à l'intégrité du corps humain, et on doit se demander au regard des évolutions récentes du droit de la santé si l'acte peut répondre aux conditions posées par l'article 16-3 du Code civil. Précisément, cela suppose de s'interroger sur la finalité de l'acte portant atteinte à l'intégrité du corps $(\mathrm{A})$, sur la qualification d'acte médical non thérapeutique (B), sur la nécessité médicale de l'acte $(\mathrm{C})$ et, enfin, sur l'exigence du consentement (D).

\section{A. LA FINALITÉ DE L’ACTE PORTANT ATTEINTE À L'INTÉGRITÉ DU CORPS : LA FINALITÉ MÉDICALE}

L'acte médical est celui qu’un homme qualifié pose en vue de guérir autrui. L’acte médical se définit par la personne qui le pratique, le médecin, mais aussi au travers de sa finalité, le diagnostic et le traitement ${ }^{7}$.

La finalité de l'acte médical justifie l'atteinte d'une part à l'intégrité corporelle du patient et permet, d'autre part, au médecin d'échapper à toute poursuite pénale. Jusqu'en 1999, le législateur légitimait cette atteinte en raison de la " nécessité thérapeutique » associée à l'acte médical. Sans cette exigence, l'acte médical recouvrait la qualification de violences volontaires et ce, indépendamment du consentement du patient.

La finalité thérapeutique visée dans la formulation initiale de l'article 16-3, exception traditionnelle du droit médical, et qui suppose une intervention sur le corps à visée diagnostique ou curative, a cédé la place à la nécessité médicale à l'occasion de la loi du 27 juillet 1999 portant création de la couverture médicale universelle ${ }^{8}$. Cette modification permet dès lors de légitimer des actes médicaux dépourvus de toute finalité diagnostique ou curative. Le professionnel médical peut ainsi réaliser des actes médicaux non thérapeutiques sans que ces derniers ne soient assimilés à une atteinte à l'intégrité corporelle du patient.

Le passage d'une nécessité thérapeutique à une simple nécessité médicale a, parconséquent,changéladonne.Eneffet,ceglissementsémantiqueapermis

\footnotetext{
$6 \quad$ Ibid., p. 174.

7 Renaut Marie-Hélène, "L'évolution de l'acte médical ", Revue de droit sanitaire et social, $1999, \mathrm{n}^{\circ} 1$, p. 45.

8 Loi n 99-641 du 27 juillet 1999 portant création d'une couverture médicale universelle.
} 
la légalisation d'actes médicaux de prévention ou de prédiction. Dès lors, la finalitédel'actemédicalnerésideplusuniquementdanslaguérisondupatient.

Pour ces actes, qualifiés d'actes médicaux non thérapeutiques, il est intéressant d'observer que les exigences légales s'inversent. En effet, leur caractère licite passe par une appréciation assouplie du critère ayant trait à la nécessité médicale et par un renforcement parallèle du consentement du sujet à l'acte. Or, en principe et en l'absence de nécessité médicale, le consentement du patient ne peut justifier à lui seul l'intervention du praticien'. En d'autres termes, comme le souligne Sophie Paricard, le consentement du sujet de droit à un acte sans nécessité médicale ne suffit pas à légitimer l'atteinte à l'intégrité corporelle. Toutefois, des assouplissements à cette règle existent et désormais avec la reconnaissance des actes médicaux non thérapeutiques, le consentement du patient devient déterminant. Dans cette perspective, c'est la liberté individuelle du sujet qui permet à l'acte de revêtir sa "nécessité médicale " telle que requise par l'article 16-3, alinéa $1^{\text {er }}$ du Code civil.

\section{B. La QUALIFICATION D’aCTE MÉdiCAL NON THÉRAPEUTIQUE}

La reconnaissance des actes médicaux non thérapeutiques a été rendue possible par la loi du 27 juillet 1999 et l'abandon de l'exigence d'une nécessité thérapeutique inhérente à l'acte médical.

Par définition, un acte médical non thérapeutique n’est pas un acte médical nécessaire. En effet, il n'existe pas de bénéfice thérapeutique direct (pour le patient) ou indirect (pour la personne, autrui ou la collectivité) ${ }^{10}$. Entrent dans cette catégorie spécifique, l'interruption non médicale de grossesse, le clonage à visée reproductive, l'administration de substances mortifères, la stérilisation contraceptive, la gestation pour autrui, l'assistance médicale à la procréation (AMP), la chirurgie esthétique. Cependant, eu égard à leur caractèrenonthérapeutique,lalicéitédecesactesestsubordonnéeàleurprise en considération expresse par le législateur.

L'interruption non médicale de grossesse a été le premier acte médical non thérapeutique reconnu par la loi en 1975. Les actes de chirurgie esthétique ${ }^{11}$,

9 Paricard Sophie, "Le consentement aux actes médicaux non thérapeutiques ", in LaUde Anne (dir.), Consentement et santé, Paris, Dalloz, 2014, p. 104.

10 Ibid., p. 105.

11 Loi $n^{\circ} 2009-879$ du 21 juillet 2009, JO nº 0167, 22 juillet 2009, p. 12184. Voir notamment l'article R. 6322-1 du Code de la santé publique : "Sont soumises aux dispositions du présent chapitre les installations où sont pratiqués des actes chirurgicaux tendant à modifier l'apparence corporelle d'une personne, à sa demande, sans visée thérapeutique ou reconstructrice ». 
la stérilisation contraceptive ${ }^{12}$ et l'assistance médicale à la procréation ${ }^{13}$ sont aujourd'hui des pratiques marquées du sceau de la licéité. À l'inverse, le clonage reproductif, l'euthanasie ou encore la gestation pour autrui demeurent des actes interdits.

Quant à la circoncision rituelle, si elle n'est visée par aucun texte, elle pourrait relever de la catégorie des actes médicaux non thérapeutiques.

Reste cependant l'exigence d'une nécessité médicale et de sa délicate justification en matière d'actes non thérapeutiques.

\section{LA NÉCESSITÉ MÉDICALE DE L’ACTE}

Deux approches permettent de justifier la nécessité médicale attachée à l'acte dépourvu de finalité thérapeutique. Pour certains auteurs, il s'agit d'une nécessité médicale "artificielle " ${ }^{14}$ liée à l'autodétermination du patient qui qualifie lui-même le caractère nécessaire de l'acte. Pour d'autres, la nécessité médicale impose la réalisation de l'acte par un médecin ${ }^{15}$ indépendamment de sa finalité thérapeutique.

\section{La qualification individuelle de la nécessité}

Traditionnellement, la nécessité de l'acte est l'apanage du médecin qui le propose à son patient. Dans cette hypothèse, la nécessité médicale doit être associée à la volonté du patient de subir l'acte afin de justifier l'atteinte à son intégrité physique ou psychique.

Âl'inverse, qu'enest-illorsquel'actemédicalestproposénon pasparl'auteur del'acte mais par son sujet? Le consentement du patient apparaîtici comme l'expressiond'unelibertépersonnelle.Parconséquent,l'autodéterminationdu patient permet dejustifierla nécessitédel'acte sansqu'il ne soitfait grand cas des motifs de sa demande.

12 Loi $\mathrm{n}^{\circ}$ 2001-588 du 4 juillet 2001 relative à l'interruption volontaire de grossesse et à la contraception, $J O \mathrm{n}^{\circ} 156,7$ juillet 2001, p. 10823.

13 Loi n ${ }^{\circ}$ 94-654 du 29 juillet 1994 relative au don et à l'utilisation des éléments et produits du corps humain, à l'assistance médicale à la procréation et au diagnostic prénatal, $J O$ $\mathrm{n}^{\circ} 175,30$ juillet 1994, p. 11060.

14 Hennion-jacquet Patricia, "Le paradigme de la nécessité médicale ", Revue de droit sanitaire et social, $\mathrm{n}^{\circ}$ 6, 2007, p. 1038.

15 Gourdon Pascal, « Une conséquence inattendue de la modification de l'article 16-3 du Code civil : la légalisation de la circoncision rituelle "médicalisée" ", Médecine \& Droit, $n^{\circ} 59,2003$, p. 44. 
Les récentes modifications en matière d'IVG illustrent parfaitement ce constat. En effet, l'exigence d'une "situation de détresse ${ }^{16}$ justifiait initialement un contrôle de la légitimité de la demande formulée par la patiente souhaitant interrompre sa grossesse.

Or depuis la loi du 4 août 2014, cette condition préalable n'est plus requise par le législateur ${ }^{17}$. Par cette modification, souhaitée par le Comité consultatif national d'éthique ${ }^{18}$, la situation de détresse, et plus encore les motivations de l'acte abortif, relèvent de l'appréciation personnelle de la femme ${ }^{19}$.

Le constat d'une absence du contrôle des motivations du patient peut être étendu à d'autres actes non thérapeutiques. Ainsi, en matière de stérilisation contraceptive, les textes en vigueur imposent uniquement l'existence d'une volonté "motivée " ${ }^{20}$ de la personne sans pour autant soumettre celle-ci à un quelconque contrôle. Concernant l'AMP, la décision appartient au couple, composé d'un homme et d'une femme, vivants et en âge de procréer. Si là encore, le Code de la santé publique exige que l'équipe médicale vérifie leur «motivation ", ce contrôle est en pratique resté " lettre morte " ${ }^{21}$. Il en est de même en matière de chirurgie esthétique, où le consentement du patient à l'opération semble, au regard de la jurisprudence, l'emporter sur l'application par le médecin du principe de proportionnalité.

Pour les actes médicaux non thérapeutiques, il apparait que la liberté individuelle suffit à justifier la nécessité de l'acte. Certains auteurs y voient là une dénaturation ${ }^{22}$ de l'exigence de la nécessité médicale, le moyen de réaliser l'acte se confondant avec sa finalité.

16 Ancien article L. 2212-1 du Code de la santé publique modifié par la loi n 2001-588 du 4 juillet 2001 - art. 1, JO, 7 juillet 2001 : « La femme enceinte que son état place dans une situation de détresse peut demander à un médecin l'interruption de sa grossesse. Cette interruption ne peut être pratiquée qu'avant la fin de la douzième semaine de grossesse ".

17 Article L. 2212-1 du Code de la santé publique (modifié par la loi n² 2014-873 du 4 août 2014 - art. 24, $J O \mathrm{n}^{\circ} 0179,5$ août 2014, p. 12949) : «La femme enceinte qui ne veut pas poursuivre une grossesse peut demander à un médecin l'interruption de sa grossesse. Cette interruption ne peut être pratiquée qu'avant la fin de la douzième semaine de grossesse ».

18 Avis CCNE no 66 du 23 novembre 2000.

19 Dans le même sens, v. affaire Cassey où la Cour des États-Unis considère que l'avortement entre dans la catégorie "des choix privés et personnels qui mettent en jeu les croyances personnelles de la femme ainsi que son destin ", citée par PARICARD Sophie, art. cit., p. 109.

20 Article L. 2123-1 al. 1 du Code de la santé publique créé par la loi $n^{\circ} 2001-588$ du 4 juillet 2001 - art. 26, JO, 7 juillet 2001 : « La ligature des trompes ou des canaux déférents à visée contraceptive ne peut être pratiquée sur une personne mineure. Elle ne peut être pratiquée que si la personne majeure intéressée a exprimé une volonté libre, motivée et délibérée en considération d'une information claire et complète sur ses conséquences".

21 Paricard Sophie, art. cit., p. 110.

22 Hennion-jacquet Patricia, art. cit., p. 1038. 


\section{La médicalisation de l'acte}

Une autre approche permet à l'acte médical non thérapeutique de répondre à l'exigence d'une nécessité médicale. En effet, comme ont pu le souligner certains auteurs, le terme est susceptible de recouvrir deux acceptions ${ }^{23}$. Selon la première d'entre elles, l'acte médical doit être "médicalement nécessaire ».

Selon la seconde approche, il est question de "la nécessité de médicaliser " un acte "socialement toléré ». Dans cette perspective, la réalisation de l'acte par un médecin permet à l'acte comme à sa nécessité de revêtir la qualification de "médicale ». Toutefois, cette interprétation de l'article 16-3 alinéa 1 du Code civil suscite la critique. Certains y voient en effet une dangereuse confusion entre les moyens de réaliser l'acte (médicalisation) et sa finalité (thérapeutique ou préventive) $)^{24}$.

En matière de circoncision rituelle, cette approche permettrait une mise en conformité de la pratique avec l'une des conditions posées par l'article 16-3 du Code civil, en réservant son exécution aux seuls médecins. Quant aux motifs confessionnels rattachés à l'acte, ils semblent, à l'instar des autres actes médicaux non thérapeutiques, laisser la place au consentement du patient.

\section{L'EXIGENCE DU CONSENTEMENT}

En principe, par application des textes sur l'inviolabilité du corps humain, les atteintes au corps sans le consentement de celui qui les subit sont illicites. Le droit civil, comme le droit pénal, sanctionnent les atteintes à l'intégrité corporelle portées à un individu sans son consentement, provenant d'un tiers ou d'un co-contractant. Les coups et blessures engagent la responsabilité pénale de leur auteur et ouvrent droit à réparation. Responsabilité délictuelle et responsabilité contractuelle le cas échéant, de l'auteur du dommage corporel peuvent être engagées.

En matière d'actes médicaux non thérapeutiques, catégorie dont peut relever la circoncision rituelle, si le contrôle des motivations du patient est assoupli, il en va différemment de son consentement. La reconnaissance légale de ces actes s'accompagne ainsi d'un encadrement légal spécifique. En outre la volonté du patient demeure déterminante et plus particulièrement lorsqu'il est question de personnes vulnérables.

23 Vialla François, "Protection de l'intégrité corporelle des enfants ", Droit et Santé, n 57, 2014, p. 2702.

24 Gourdon Pascal, art. cit., p. 44. 


\section{Le renforcement de la protection du consentement pour les actes médicaux non thérapeutiques}

Les actes médicaux non thérapeutiques font l'objet d'un régime spécial en matière de consentement. Ces dispositions législatives propres portent sur l'information du patient ainsi que sur le recueil de son consentement.

En matière d'actes médicaux non thérapeutiques, l'information doit être exhaustive afin que la décision du patient soit prise en connaissance de cause. L'information porte ainsi sur toutes les conséquences possibles de l'opération et sur l'ensemble des risques même exceptionnels encourus par la personne.

À titre d'exemple, pour ce qui est de l'IVG, l'information doit porter sur les « méthodesmédicalesetchirurgicalesd'interruptiondegrossesseetdesrisques et des effets secondaires potentiels ${ }^{25}$. Concernant la stérilisation contraceptive, le médecin doit informer des " risques médicaux » et des " conséquences de l'intervention $»^{26}$. En matière de chirurgie esthétique, il lui est fait obligation d'informer le patient « des risques et des éventuelles conséquences et complication $"{ }^{27}$. Enfin, pour ce qui est de l'assistance médicale à la procréation, le législateurprécisequel'informationdoitportersur « lespossibilités derisques etd'échecdestechniques d'AMP, deleurseffets secondaireset deleurs risques à court et long terme, ainsi que de leur pénibilité et des contraintes qu'elles peuvent entrainer $»^{28}$.

Àcetteinformationspécifique,s'ajoutela remised'undocumentécritparle médecin (« dossier guide » pour l'IVG et l'AMP, « dossier d'information écrit » pour la stérilisation, « devis détaillé » pour la chirurgie esthétique).

Un régime spécial est pareillement prévu en matière de recueil du consentement. La première spécificité réside dans la formalisation du consentement par le biais d'un écrit. Ainsi, une confirmation écrite de la volonté de la personne est exigée en cas d'IVG ${ }^{29}$, de stérilisation à visée contraceptive ${ }^{30}$, d'AMP $^{31}$. En matière de chirurgie esthétique, cet écrit prend la forme d'un devis détaillé ${ }^{32}$.

Àl'exigenced'unconsentementécrit,s'ajoutel'aménagementd'undélaide réflexion permettant d'éviter l'écueild'une décision précipitée (une semaine pour I'IVG, quatre mois pour la stérilisation contraceptive, un mois pour une $A M P$, quinze jours minimum pour un acte de chirurgie esthétique).

\footnotetext{
25 C. santé publ., art. L. 2212-3.

26 C. santé publ., art. L. 2123-1, al. 4.

27 C. santé publ., art. L. 6322-2.

28 C. santé publ., art. L. 2141-10.

29 C. santé publ., art. L. 2212-5.

30 C. santé publ., art. L. 2123-1, al. 4.

31 C. santé publ., art. L. 2141-10.

32 C. santé publ., art. L. 6322-2.
} 
Ces dispositions propres à la réalisation de l'acte médical non thérapeutique en garantissent la légalité et attestent de l'importance de la volonté du patient.

\section{Le renforcement de l'autonomie décisionnelle des mineurs}

Par principe, le mineur ne peut consentir à l'acte médical. En effet, au regard des dispositions de l'article L. 1111-2 du Code de la santé publique, ce droit est exercé par les titulaires de l'autorité parentale. Il peut néanmoins participer à la prise de décision dans la mesure où son degré de maturité le permet.

Or il apparait qu'en matière d'actes médicaux non thérapeutiques, le mineur bénéficie d'une " autonomie décisionnelle inédite ${ }^{33}$ lui permettant de consentir lui-même à l'acte.

Deux situations doivent cependant être distinguées selon que l'acte en question revêt ou non un caractère urgent.

Lacte médical urgent est entendu comme celui qui ne peut être retardé à la majorité de l'enfant. Dès lors, la liberté personnelle du mineur suffit à autoriser la réalisation de l'acte. L'exemple est donné en matière d'IVG, où l'absence de consentement des titulaires de l'autorité parentale n'empêche pas l'intervention. La mineure devra néanmoins être accompagnée dans sa démarche par une personne majeure de son choix ${ }^{34}$.

Si l'acte n'est pas urgent (c'est-à-dire qu'il peut être retardé jusqu'à la majorité de l'enfant), il semble que ni le mineur ni les titulaires de l'autorité parentale ne puissent consentir à l'acte. Dans cette hypothèse, il est nécessaire d'attendre la majorité de la personne concernée par l'acte, seul moment où il pourra valablement consentir à l'acte. Cette solution est celle prévalant en matière de stérilisation contraceptive.

Toutefois, comme le fait observer Sophie Paricard, à propos de la circoncision "L'attente de la majorité de l'enfant se heurte au souci de préserver une relation équilibrée entre droit et religion mais surtout entre liberté de conscience de l'enfant et respect de l'éducation religieuse donnée par ses parents. Il est peutêtre plus opportun de préserver une coutume religieuse qui n'est pas invalidante et dont les écueils, très marginaux, ont toujours été sanctionnés par le droit ${ }^{35}$.

33 Paricard Sophie, art. cit., p. 111.

34 C. santé publ., art. L. 2212-7.

35 Paricard Sophie, art. cit., voir p. 116. 


\section{LA RELIGION DE L'ENFANT : UNE PRÉROGATIVE PARENTALE}

Le choix de la religion de l'enfant relève des attributs de l'autorité parentale. Bien que le législateur français n’ait pas expressément prévu à qui revient la tâche de choisir la religion de l'enfant ${ }^{36}$, doctrine et jurisprudence se sont toujours accordées pour considérer que l'éducation religieuse est un des aspects de l'éducation en général et dès lors qu'elle relève des devoirs et prérogatives parentaux. La Convention internationale des droits de l'enfant (CIDE) en fait l'un des droits et devoirs des parents que les États parties à la Convention doivent s'engager à respecter. L'article $14-2^{\mathrm{e}}$ dispose que "Les États respectent le droit et le devoir des parents de guider celui-ci dans l'exercice du droit à la liberté de pensée, de conscience et de religion d'une manière qui corresponde au développement de ses capacités ». Il faut ici remarquer que la même Convention place en tête le droit de l'enfant à la liberté de pensée, conscience et religion, ce qui est susceptible de créer une contradiction entre les droits parentaux (issus donc de l'autorité parentale) et la reconnaissance au mineur de certains droits. En effet, dans le cadre de l'autorité parentale, le mineur est seulement objet de droit et n'est titulaire d'aucun droit. Or certains droits reconnus aux mineurs via la CIDE s'articulent difficilement avec les prérogatives parentales, ce qui permet de douter de leur effectivité ${ }^{37}$.

S'il appartient donc aux parents de choisir l'orientation religieuse de l'enfant, toutefois cette prérogative ne s'exerce pas sans limite. Ainsi et de manière générale, les titulaires de l'autorité parentale ne doivent pas mettre en danger la santé, la sécurité ou la moralité du mineur, par leur conformité aux impératifs religieux, ou encore compromettre les conditions de son éducation ou de son développement par cette obéissance aux dogmes et pratiques religieux. Si tel est le cas, il faut protéger l'enfant et le protéger alors contre ses parents ${ }^{38}$.

Le juge doit concilier la liberté de croyance et de religion dont disposent les parents à l'égard de l'enfant ainsi que leur liberté éducative qu'il n'a pas en principe à contrôler et parallèlement assurer de manière efficace la protection

36 Comme le souligne Boulanger François ("Autorité parentale et formation religieuse des mineurs, essai comparatif ", Droit de la famille, $\mathrm{n}^{\circ} 11,2013$, étude 14), "il a été assez peu remarqué que le mot religion ne soit pas comme tel, mentionné, sauf une allusion feutrée dans le Code de procédure civile à propos de l'assistance éducative sur le respect des convictions religieuses ou philosophiques du mineur et de sa famille ».

37 Bonfils Philippe, Gouttenorre Adeline, Le droit des mineurs, Paris, Dalloz, coll. Précis, $2^{e}$ éd., 2014, n 541, p. 343 ; v. également, Terré François, Fenouillet Dominique, Droit civil. Les personnes, Paris, Dalloz, coll. Précis, $8^{e}$ éd., 2012, n 374 et s., p. 341 et s.

38 Fortier Vicente, "La protection de l'enfant contre les pratiques religieuses de ses parents », in Fortier Vincente, LEBEL-Grenier Sébastien (dir.) La vulnérabilité et le droit : entre autonomie et paternalisme, Sherbrooke, éditions RDUS, 2010, p. 25-44. 
de cette personne particulièrement vulnérable qu'est l'enfant. Dans le cas précis de la circoncision rituelle, la liberté religieuse dont disposent les parents au titre de l'autorité parentale heurte le principe d'inviolabilité du corps humain et la liberté religieuse reconnue à l'enfant.

Comment résoudre le double conflit de normes que révèle la circoncision rituelle, celui résultant de l'opposition entre impératif religieux et impératif juridique, et celui tenant aux dispositions protégeant le corps humain et les attributs relevant de l'autorité parentale?

Une partie de la réponse, ou du moins certaines voies de règlement des conflits sont apportées par la jurisprudence civile. La circoncision rituelle se retrouve au cœur du débat judiciaire principalement dans deux situations, s'inscrivant dans un conflit parental sur l'éducation religieuse du mineur : d'une part, lorsque l'un des parents demande au juge l'autorisation de procéder à la circoncision sur un enfant ; d'autre part, lorsque l'un des parents a fait circoncire l'enfant sans avoir requis au préalable le consentement de l'autre parent.

Le traitement judiciaire de l'acte de circoncision rituelle va permettre de poser les bases de son accomplissement dans des conditions respectueuses du choix parental et de la liberté religieuse des parents.

Loin d'ignorer la circoncision rituelle ou de manifester une indifférence à son égard, le juge civil donne, ici, leur pleine mesure aux règles de l'autorité parentale pour aménager le cadre de l'accomplissement de la circoncision rituelle et tracer les limites de la liberté religieuse des parents à l'égard de l'enfant. En réalité, il s'agit moins ici de protéger l'enfant que de protéger les droits des parents. En effet, et curieusement, la notion d'intérêt de l'enfant n'est quasiment jamais évoquée, du moins de manière explicite. Or, l'autorité parentale est une fonction qui est finalisée car les droits et devoirs des parents se déploient dans l'intérêt de l'enfant. Cette notion constitue la pierre angulaire de l'autorité parentale et du droit de l'enfance en général, la Convention internationale relative aux droits de l'enfant utilise l'expression " d'intérêt supérieur de l'enfant " 39 . Mais si la question de l'intérêt de l'enfant n'est pas posée, c'est sans doute parce que le juge civil est le plus souvent placé devant " le fait accompli ». Il est permis de se demander du reste où se situe l'intérêt de l'enfant : dans le respect de la tradition religieuse de sa famille, gage de son intégration dans sa communauté ou bien dans la protection de son intégrité corporelle mais également de sa propre liberté religieuse ${ }^{40}$ ?

39 Sur l'applicabilité directe de la CIDE, voir les développements de Bonfils Philippe, Gouttenorre Adeline, op. cit., n ${ }^{\circ} 60$ et s, p. 34 et s.; et dans le même ouvrage, à propos de l'intérêt supérieur de l'enfant, $\mathrm{n}^{\circ} 92$ et s., p. 61 et s. 


\section{A. La QUALIFICATION DE LA CIRCONCISION RITUELLE}

\section{La circoncision rituelle : une atteinte à l'intégrité physique}

La qualification de la circoncision rituelle par la jurisprudence en tant qu'atteinte à l'intégrité physique du corps de l'enfant est parfois évoquée. Tel est le cas, par exemple, de la Cour d'appel de Lyon, saisie par le père d'une demande d'autorisation de faire circoncire l'enfant ${ }^{41}$. Dans une décision récente, la Cour d'appel de Poitiers considère que la circoncision est " une atteinte directe au corps de l'enfant petit » soulignant " le caractère définitif et symbolique de cette opération ${ }^{42}$. Telle était déjà la position de la Cour d'appel de Rennes ${ }^{43}$ dans une affaire ancienne opposant un père algérien et une mère française catholique, qualifiant la circoncision (en l'espèce « imposée aux enfants dans des conditions menaçant leur équilibre "), de "voie de fait puisqu'il y a atteinte à l'intégrité physique ", relevant qu' "il est inexact que la pratique soit courante en France ».

Deux décisions doivent ici être signalées qui, bien qu'elles s'inscrivent dans un contexte particulier (enfants faisant l'objet d'une mesure de placement), illustrent un certain durcissement de principe, à tout le moins une prise de distance, à l'égard de la circoncision. La première affaire est un arrêt de la Cour d'appel de Lyon ${ }^{44}$. La mère titulaire de l'autorité parentale et dont les enfants étaient placés, demandait que ceux-ci soient instruits dans la religion musulmane (le père était d'accord) et s'interrogeait sur une éventuelle circoncision. Si la cour décide que le service gardien doit mettre en place un enseignement à la culture et à la religion musulmane, toutefois sur le point de la circoncision,

41 CA Lyon, 25 juillet 2007, JurisData n 2007-346158 : "S'agissant d'une atteinte à l'intégrité physique de l'enfant, cette décision grave ne peut être prise que d'un commun accord entre les parents et avec le consentement de l'enfant, âgé de 11 ans ; en l'absence de consentement tant de la mère que de l'enfant qui a exprimé à de maintes reprises son opposition à une telle opération, le rejet de la demande s'impose ». v. également, CA Nancy, 5 octobre 2009, JurisData $\mathrm{n}^{\circ}$ 2009-023366 confirmant l'interdiction faite au père de procéder à la circoncision de l'enfant ; dans cette affaire et se fondant sur l'article 3732-6 du Code civil les conseillers nancéens rappellent que «la protection et l'éducation de l'enfant qui doit aussi s'entendre comme l'éducation religieuse, sont les raisons d'être de l'autorité parentale. Ainsi compte tenu de l'importance que revêt toute décision en matière religieuse, il n'est pas envisageable que cette décision soit prise sans l'accord des deux parents qui exercent en commun l'autorité parentale». Également, CA Riom, 17 avril 2007, JurisData n ${ }^{\circ}$ 2007-336812.

42 CA Poitiers, 11 mai 2011, JurisData, inédit, jugeant dès lors que cette opération ne pouvait pas être décidée par le père seul sans consultation et accord exprès de la mère.

43 CA Rennes, 23 janvier 1991, cité par ChoAin Christine, note sous Cass. $1^{\text {re }}$ civ., 26 janvier 1994, D. 1995, jurisprudence p. 226. Dans cette affaire, le père s'est vu retirer son droit de visite.

44 CA Lyon, 31 mai 2011, JurisData, inédit. 
elle considère qu'un bilan auprès d'un établissement hospitalier devra être réalisé "permettant d'apprécier la compatibilité d'une éventuelle circoncision avec l'état de santé actuel des garçons, leur degré de discernement de cet acte et de ses conséquences ainsi que du retentissement psychologique prévisible ». La cour rappelle, de surcroît, que " la Convention internationale des droits de l'enfant pose comme principe que tout enfant a le droit à son intégrité physique et à faire le choix de la religion qu'il entend pratiquer, les titulaires de l'autorité parentale ayant quant à eux le droit de faire élever leurs enfants dans la connaissance de leurs religions communes ». La solution semble donc faire une distinction entre, d'une part, la circoncision qui porte atteinte à l'intégrité physique de l'enfant et à son libre choix d'une religion et, d'autre part, la prérogative parentale. Est-ce à dire que le droit des parents d'élever leurs enfants dans la religion de leur choix bute contre une limite, celle de ne pas porter atteinte au corps de l'enfant et à sa propre liberté de choix ?

Allant plus loin encore, une ordonnance rendue en 2002, par le tribunal de grande instance de Laval ${ }^{45}$, considère que "la circoncision pratiquée comme un rite religieux porte volontairement atteinte à l'intégrité physique et constitue manifestement une forme de violence sexuelle ". Rappelant la Convention internationale des droits de l'enfant et particulièrement son article 34 qui fait obligation aux États parties de protéger l'enfant contre toutes les formes de violence sexuelle, le juge des enfants de Laval ajoute que « cet enfant, âgé de six ans, n'est pas en mesure en raison de son âge, de donner un quelconque consentement à cet acte chirurgical qui provoquera des conséquences physiques irrémédiables ainsi que selon plusieurs spécialistes des difficultés dans sa vie sexuelle future ». Il faut souligner ici que le juge a interdit la circoncision rituelle alors que les deux parents y étaient favorables. Certes le contexte de l'affaire était particulier ; il s'agissait d'un enfant qui avait été confié aux services d'aide sociale à l'Enfance. Cependant, les parents conservent l'autorité parentale durant ce placement, ce qui en l'état actuel de la jurisprudence sur la circoncision et considérant leur assentiment, aurait dû conduire à permettre l'intervention. Toutefois ce qui est particulièrement intéressant ici est cette interdiction posée formellement, le juge qualifiant la circoncision rituelle de violence sexuelle.

\section{La circoncision rituelle : un acte non usuel de l'autorité parentale}

$\mathrm{Si}$, dans les décisions, la référence à l'atteinte à l'intégrité physique n'est pas toujours énoncée, il n'en demeure pas moins que dans l'immense majorité des cas, la circoncision rituelle est qualifiée du point de vue des actes de l'autorité

45 TGI Laval, juge des enfants, 16 avril 2002, AJ Famille 2002, p. 222, note. 
parentale, d'acte grave, ou d'acte important et unique dans la vie de l'enfant, d'acte non usuel de l'autorité parentale ${ }^{46}$.

Cette qualification d'acte grave ou non usuel de l'autorité parentale n'est pas neutre : elle emporte des conséquences en termes de responsabilité parentale et va permettre au juge de poser le cadre de l'accomplissement du rite religieux.

Le Code civil ne définit pas ce qu'il faut entendre par acte usuel ou non usuel de l'autorité parentale. Les actes usuels de l'autorité parentale bénéficient d'une présomption d'accord selon l'article 372-2 du Code civil. S'agissant des actes sur la personne de l'enfant, les actes de soins courants sont certainement des actes usuels ${ }^{47}$. En revanche, tel n'est pas le cas de l'éducation religieuse en général ${ }^{48}$ et de la circoncision rituelle en particulier.

Àcetégard,uneaffaireanciennetraitéeparletribunaldegrandeinstancede Paris le 6 novembre $1973^{49}$ est riche d'enseignements sur la difficulté à distinguer ce qui relève de l'opération relativement bénigne et médicalement nécessaire (opération du phimosis) pour laquelle, du reste, le consentement des deux parents n'est pas requis et de la circoncision rituelle qui, à l'inverse, nécessite le double consentement. Le tribunal était saisi d'une demande du père mettant en cause la responsabilité du médecin qui ne s'était pas assuré de son consentement à propos de la circoncision de ses trois garçons. Les faits étaient, à tout le moins, ambigus : une mère avait fait opérer le même jour ses trois fils, âgés respectivement de sept, six et cinq ans, d'un phimosis. La question se posait de savoir si le médecin pouvait procéder à cette opération sur les enfants à la seule demande de la mère. Mais les faits étaient troublants: les trois garçons avaient été opérés le même jour. Les parents tout comme vraisemblablement le médecin étaient de confession juive. Cependant, seule la mère était pratiquante. Le père était opposé à la circoncision rituelle. Il avait, donc, assigné le médecin en réparation

46 CA Rennes, 4 avril 2005, JurisData n 2005-292420 ; CA Paris, 29 septembre 2000, D. 2001, p. 1585, note Duvert Cyrille.

47 À propos de la distinction acte usuel/acte important ou non usuel, v. notamment les exemples in Bonfils Philippe, Gouttenorre Adeline, op. cit., $\mathrm{n}^{\circ} 561$ et s., p. 351 et s. ; v. également Terré François, Fenouillet Dominique, op. cit., n 429, p. 426.

48 Les décisions concernant l'éducation religieuse de l'enfant relèvent toujours de la catégorie des choix importants relatifs à la vie de l'enfant et qui, par conséquent requiert l'assentiment des deux parents. Par exemple, CA Nîmes, 20 juin 2012, JurisData, inédit, condamnant un père qui avait organisé la cérémonie de la bar-mitsva de l'enfant âgé de douze ans lors d'un voyage en Israël sans que la mère n'en ait été informée. Si la mère avait accepté que l'enfant reçoive une éducation religieuse, la cour d'appel constate que «cet accord ne concernait que la participation à une éducation religieuse non nécessairement suivi d'un engagement suffisamment important et sérieux pour entraîner la conversion à cette religion »; CA Montpellier, 30 mai 1988, JurisData $\mathrm{n}^{\circ}$ 11583, selon laquelle une mère ne peut pas, de sa propre initiative, retirer un enfant d'un établissement scolaire religieux, choisi antérieurement par les deux parents, pour le placer dans un établissement laïque.

49 TGI Paris, 6 novembre 1973, Gaz. Pal. 1974, 1, p. 299, note Barbier P. 
de son préjudice moral propre et en réparation du préjudice physique et moral éprouvé par ses fils. Il considérait qu' " une telle intervention ne peut être considérée comme un acte usuel en France, et qu'en raison de son caractère particulier, l'ordre opératoire de la mère était insuffisant ». Evidemment il insistait sur le " caractère insolite " de ces interventions, les trois frères présentant au même moment un problème de santé identique nécessitant une opération d'urgence. Il faisait état de l'atteinte irréversible à l'intégrité corporelle de ses fils avec toutes les conséquences psychologiques, morales et sociales que cette marque physique comportait. Tout le problème résidait dans la qualification de ces interventions : s'agissait-il d'actes médicaux (phimosis) ou de circoncisions rituelles? Après expertise, le tribunal considéra que deux des enfants présentaient bien un phimosis et que le médecin n'était pas tenu avant de pratiquer une opération relativement bénigne et médicalement nécessaire de recueillir d'autre autorisation que celle de la mère, rien ne lui laissant supposer un désaccord parental. Le tribunal ajouta que la situation aurait pu être différente s'il s'était agi d'une circoncision rituelle. Mais en l'espèce c'est "par une simple coöncidence " que les convictions religieuses des parents différaient, la mère étant pratiquante et le père étant opposé à la circoncision. Quant au plus jeune des enfants, le cas était plus litigieux, les experts n’ayant pu établir formellement la nécessité de l'intervention. Le tribunal considéra, alors, qu'il y avait lieu de présumer que l'enfant était dans le même cas que ses frères, " ce qui paraît correspondre aux lois de l'hérédité physiologique et que l'intervention s'imposait pour lui de la même façon ». Il ne s'agissait donc pas de circoncision rituelle de sorte que le seul consentement de la mère suffisait.

\section{B. LA CIRCONCISION RITUELLE : UNE CO-DÉCISION PARENTALE}

Tirant les conséquences de la qualification juridique de la circoncision rituelle relativement à l'autorité parentale, le juge civil pose les conditions de sa réalisation, signifiant par là même aux parents les limites à observer dans l'exercice de leurs prérogatives.

Précisément, s'agissant d'un acte non usuel de l'autorité parentale, l'accord des deux parents est requis pour procéder à la circoncision de l'enfant ${ }^{50}$. C'est pourquoi, du reste, les juges refusent de se substituer au parent réticent, lorsqu'ils sont saisis d'une demande d'autorisation de faire procéder à la

50 CA Paris, 13 septembre 2000, JurisData n 2000-125029 ; CA Rennes, précit. ; CA Orléans, 14 mars 2006, JurisData n ${ }^{\circ}$ 2006-310764. Contra: CA Aix-en-Provence, 4 mars 2004, JurisData n ${ }^{\circ}$ 2004-238032 (concernant deux époux de confession musulmane). 
circoncision $^{51}$. On ajoutera à cela que la situation est bien embarrassante. Comment juger de l'intérêt de l'enfant (puisque la notion doit être centrale) à être circoncis (ou baptisé) et donc décider, in fine, de l'orientation religieuse de cet enfant, alors qu'aucun critère religieux ne peut intervenir dans le choix du juge? Il peut arriver que les parents se soient mis d'accord sur l'intervention selon certaines modalités. Le juge constate alors cet accord ${ }^{52}$.

En d'autres termes, et d'une part, la circoncision ne sera interdite qu'à partir du moment où l'assentiment des deux parents n'est pas obtenu. D'autre part, et en conséquence, une circoncision pratiquée en violation de cette règle de codécision emportera sanction du parent et/ou du praticien ayant enfreint cette obligation.

C'est ainsi que la carence de l'un des parents est un motif grave de nature à entraîner la modification du droit de visite et d'hébergement, voire la suppression de l'un d'eux ou des deux pour le parent fautif ${ }^{53}$. Dans l'hypothèse où l'autorité parentale était exercée conjointement, celle-ci peut être confiée à titre exclusif à l'un seul des parents lorsque le manquement est qualifié de " particulièrement grave ", comme a pu en décider la Cour de Lyon dans une décision récente $^{54}$. En l'espèce, il s'agissait d'un père qui s'était rendu en Algérie avec ses enfants et avait fait procéder à la circoncision du plus jeune fils âgé de trois ans, sans en informer la mère tout en connaissant parfaitement son refus d'élever les enfants dans la religion musulmane. La circoncision fut pratiquée dans de très mauvaises conditions. La Cour faisant droit à la demande de la mère

51 Par exemple, CA Aix-en-Provence, 24 septembre 2013, n 2013/468, JurisData, inédit ; CA Colmar, 8 janvier 2014, $\mathrm{n}^{\circ}$ 12/03864, JurisData, inédit.

52 CA Nîmes, 8 janvier 2014, JurisData, inédit (accord des parents sur une circoncision à compter du cinquième anniversaire de l'enfant, en présence de la mère et sous contrôle médical).

53 CA Poitiers, 21 novembre 2000, JurisData n 2000-146397 ; CA Orléans, 14 mars 2006, JurisData $\mathrm{n}^{\circ}$ 2006-310764 : dans cette affaire, les circonstances étaient troubles, le père ayant vraisemblablement tenté de circoncire l'enfant lui-même, une lésion importante avait affecté le prépuce de celui-ci, de surcroît psychologiquement traumatisé. Les juges ont considéré qu'à tout le moins la négligence du père constituait un motif grave au sens de l'article 373-2-1 du Code civil imposant de revoir les modalités d'organisation du droit de visite paternel ; CA Riom, 17 avril 2007, JurisData n ${ }^{\circ}$ 2007-336812 ; CA Lyon, 10 janvier 2011 : le père s'était rendu en Algérie et avait fait circoncire son fils âgé de trois ans alors que la mère refusait d'élever l'enfant dans la religion musulmane. Lors de son retour en France, l'enfant présentait une infection majeure. La cour qualifie le manquement du père aux règles de l'autorité parentale conjointe comme étant particulièrement grave et dès lors confie l'exercice de l'autorité parentale à titre exclusif à la mère. L'arrêt interdit au père d'amener les enfants dans une communauté religieuse et/ou dans une école confessionnelle, interdit la sortie du territoire national et même supprime le droit de visite et d'hébergement le mercredi dans la mesure où il s'agit pour lui d'un moyen d'emmener les enfants dans une mosquée.

54 CA Lyon, 10 janvier 2011, JurisData, inédit. 
lui confie l'exercice de l'autorité parentale à titre exclusif, modifie également le droit de visite et d'hébergement du père en supprimant le mercredi après-midi à la demande de la mère (afin que le père ne puisse pas emmener les enfants à la mosquée) et condamne le père à verser 1000 euros de dommages-intérêts à la mère en réparation du préjudice subi par elle du fait du comportement fautif du père.

Il en est de même dans le litige traité par la Cour de Toulouse, le 13 octobre $2014^{55}$. Les parents (séparés) exerçaient conjointement l'autorité parentale. La mère ayant fait pratiquer la circoncision sur l'enfant lors de vacances au Gabon, à l'insu du père, celui-ci avait déposé plainte pour violences volontaires sur mineur de six ans. Le juge aux affaires familiales, saisi par le père, lui octroie l'exercice exclusif de l'autorité parentale. La Cour d'appel de Toulouse confirme le jugement " compte tenu du contexte lourd dans lequel évoluent la mère et l'enfant, et l'absence de reprise du dialogue entre les parents ", insistant sur le caractère exceptionnel de l'exercice unilatéral de l'autorité parentale qui ne peut résulter que de motifs graves.

En outre, le fait de faire pratiquer la circoncision sans l'accord, en l'espèce du père de l'enfant, et sans raison médicale est considéré comme un grief pouvant être imputé à faute à l'épouse et dès lors justifier le prononcé du divorce à ses torts ${ }^{56}$.

Quant au médecin qui pratiquerait une circoncision sans s'assurer du double consentement, il verrait sa responsabilité mise en cause : en agissant ainsi, il ferait preuve d'une légèreté blâmable ${ }^{57}$.

Cette quasi-unanimité à l'égard de la circoncision quant à son caractère de gravité et à la nécessité d'une co-décision est parfois rompue au détour de quelques décisions dont la motivation est, à tout le moins, étonnante. Ainsi de cet arrêt rendu par la Cour de Versailles ${ }^{58}$ : la mère avait fait circoncire l'enfant sans prévenir le père qui était opposéà cette pratique. Les juges considèrent que cettecirconcision, malgréle désaccord du père, nejustifie pas à elle seule lechangementderésidencedel'enfant,secontentantdepréciseràlamère « de ne pas renouveler à l'avenir ce type d'initiative, constitutive d'une atteinte aux droits de l'autre parent ». À propos d'un père ayant fait circoncire son fils sans

55 CA Toulouse, 13 octobre 2014, JurisData, inédit.

56 CA Paris, 5 novembre 2013, $n^{\circ}$ 12/18336, JurisData, inédit : "Ces faits qui démontrent l'absence totale de considération de $\mathrm{M}^{\mathrm{me}} \mathrm{L}$. envers son mari, constituent une violation grave ou renouvelée des obligations du mariage qui rend intolérable le maintien du lien conjugal ». On notera que dans cette affaire, il s'agit du seul grief.

57 CA Paris, 29 septembre 2000, D. 2001, p. 1585. À propos de cette affaire, la cour a également retenu la responsabilité du père de l'enfant ayant " profité de son droit d'hébergement pour prendre la grave décision de faire procéder, à des fins rituelles, à l'opération sans avoir recueilli l'assentiment de la mère ". 
en prévenir la mère, les juges de Lyon estiment « déplorable que le père ait cavalièrement décidé d'y procéder de sa propre initiative " ${ }^{59}$.

Par l'effet de sa judiciarisation, la circoncision rituelle entre dans le champ du droit. En posant les conditions de sa réalisation, le juge civil signifie par là même dans quelles conditions elle peut valablement être effectuée. En d'autres termes, la circoncision n'est pas interdite dès lors que ce cadre est respecté. La circoncision, de fait, ne sera interdite qu'à partir du moment où les conditions risquent de ne pas être respectées. Par conséquent, ce n'est pas l'acte en luimême qui est sanctionné mais les éventuelles circonstances de son accomplissement, en ce que de manière générale, elles contreviennent aux règles sur la co-décision parentale.

Ces décisions signifient, a contrario, que si les consentements des deux parents sont réunis, alors rien ne s'oppose à la circoncision de l'enfant. Dès lors, on peut considérer que l'impératif religieux a supplanté l'impératif juridique. Le conflit entre impératif religieux et impératif juridique se résout au profit du premier. Le rite l'emporterait sur la règle de droit d'ordre public à laquelle il contrevient pourtant. Dans cette concurrence entre les deux ordres normatifs, est privilégiée ici une conception procédurale du droit, le juge vérifiant si les conditions posées à l'accomplissement de l'acte sont ou non remplies. Cependant, il est possible de s'en tenir à une stricte analyse juridique, qui gommerait l'aspect religieux du débat. Dans cette perspective, la primauté serait donnée aux prérogatives parentales en matière de choix religieux, à la liberté religieuse et éducative des parents à l'égard de leurs enfants sur d'autres règles telle que celle posée par l'article 16-3 du Code civil.

\section{LA RÉPARATION DES CONSÉQUENCES DOMMAGEABLES DE L'ACTE DE CIRCONCISION}

La circoncision rituelle ne peut pas être considérée, médicalement, comme un acte "bénin ». En témoignent quelques affaires qui montrent la gravité des atteintes pouvant affecter l'enfant : nécrose du gland, hémorragie et, dans les cas les plus graves, décès de l'enfant à la suite de l'anesthésie pratiquée sur lui.

Lorsqu'un accident survient, lors de la circoncision, analysée comme un fait juridique, suivront des conséquences, en termes de responsabilité civile et donc de réparation.

59 CA Lyon, 7 mai 2009, JurisData n ${ }^{\circ}$ 2009-020508; mais il est vrai que dans cette affaire, le fils aîné " semblait " avoir déjà subi cette intervention sans opposition de la mère. 
En $1994^{60}$, la responsabilité d'un médecin, stomatologiste, habilité par les autorités religieuses à pratiquer les circoncisions rituelles en qualité de mohel, a été retenue, l'enfant ayant été victime d'une nécrose irréversible. La Cour d'appel de Paris ${ }^{61}$ sur cette affaire avait pointé la faute d'imprudence et de négligence : la cour considère que sa profession de stomatologue ne lui donnait aucune compétence pour apprécier correctement la suite à donner en cas de complications. De plus, et alors même que les parents s'apprêtaient à conduire l'enfant à l'hôpital, ce médecin les en avait dissuadés. Dès lors " ayant décidé de surveiller seul l'évolution de la complication survenue à la suite de la circoncision, ce médecin doit assumer l'entière responsabilité des conséquences dommageables résultant de son erreur d'appréciation du risque encouru et de son défaut de surveillance ".

En $1989^{62}$, la Cour de cassation a eu à connaître d'une affaire dans laquelle un rabbin, mohel diplômé, avait pratiqué une circoncision qui avait entraîné une nécrose du tiers de la verge de l'enfant ayant nécessité l'ablation des deux tiers des tissus. Les parents de l'enfant avaient assigné le mohel et son assureur en paiement de dommages-intérêts. La Cour d'appel de Douai avait rejeté la demande. La Cour de cassation rejeta le pourvoi considérant que l'obligation s'imposant au mohel, tant en ce qui concerne l'intervention que le contrôle postopératoire, s'analysait en une obligation de moyens et dès lors la responsabilité ne pouvait être retenue que sur le fondement d'une faute en relation causale avec l'accident. Or aucun manquement en relation avec les préjudices subis n'était établi.

Quant à la légèreté blâmable dont fait preuve le médecin qui pratique une circoncision sans s'assurer de l'accord des deux parents, elle est également passible de dommages-intérêts. Ainsi dans l'affaire précitée de la Cour d'appel de Paris en date du 29 septembre 2000, le médecin et le père de l'enfant ont été condamnés à payer 50000 francs au titre du préjudice moral de la mère et 100000 francs au titre du préjudice tant moral que corporel de l'enfant.

En outre, la responsabilité de l'hôpital au sein duquel la circoncision a été pratiquée, peut être engagée au prix d'un raisonnement spécifique, s'agissant d'un acte à finalité cultuelle. L'affaire est la suivante :

Le 9 avril 1980, un jeune enfant âgé de cinq ans est hospitalisé dans un établissement public, pour y subir une circoncision rituelle. Au cours de l'intervention, l'enfant est victime d'un arrêt cardiaque. Il sombre alors dans un coma qui va durer une année. Puis il décède.

60 Cass. $1^{\text {re }}, 6$ déc. 1994, D. 1995, inf. rap., p. 38.

61 CA Paris, 12 février 1992, D. 1993, somm. p. 27, obs. Penneau Jean.

62 Cass. $1^{\text {re }}, 18$ mai 1989, JurisData ${ }^{\circ} 1989-002212$. 
Après avoir vainement emprunté la voie répressive, la mère de l'enfant va se tourner vers les juridictions administratives afin de voir condamner l'hôpital à l'indemniser du préjudice subi du fait du décès de l'enfant. Le tribunal administratif de Marseille ${ }^{63}$ fait droit à sa demande et condamne l'hôpital au versement de 60000 francs. Selon les premiers juges, " alors qu'aucune faute n'a pu être établie dans l'organisation ou le fonctionnement d'un hôpital à l'occasion d'actes anesthésiques ou d'actes chirurgicaux, la survenance d'un accident ayant entraîné la mort d'un enfant à l'occasion d'une opération bénigne révèle une faute dans l'organisation ou le fonctionnement du service ". Il faut noter que la mère de l'enfant invoquait, notamment, la faute de l'anesthésiste qui se serait absenté au cours de l'intervention. Toutefois et selon les rapports d'expertise, celle-ci n'avait été établie, ni au cours de l'opération, ni à l'occasion des soins postopératoires.

Le tribunal administratif se plaçait ainsi sur le terrain de la faute présumée pour fonder la responsabilité de l'hôpital, solution qui, si elle avait le mérite "de faire coïncider la justice et le bon sens " ${ }^{64}$, était difficilement applicable en l'espèce. En effet, la théorie de la faute présumée n'a été retenue en jurisprudence que pour des actes bénins. Même à considérer que la circoncision entre dans cette catégorie, une anesthésie générale, en revanche, ne peut recevoir cette qualification, anesthésie qui, selon les experts, avait été à l'origine de l'accident cardiaque de l'enfant.

Saisie sur requête de l'hôpital, la Cour administrative de Lyon ${ }^{65}$ condamna l'hôpital, en appliquant fidèlement la solution précédemment dégagée par la jurisprudence administrative lors de l'arrêt Bianchi ${ }^{66}$, substituant au régime de responsabilité pour faute présumée, un régime de responsabilité sans faute fondé sur le risque : "Considérant que lorsqu'un acte médical nécessaire au diagnostic ou au traitement du malade présente un risque dont l'existence est connue mais dont la réalisation est exceptionnelle et dont aucune raison ne permet de penser que le patient y soit particulièrement exposé, la responsabilité du service public hospitalier est engagée si l'exécution de cet acte est la cause directe de dommages sans rapport avec l'état initial du patient comme avec l'évolution

63 TA Marseille, 25 avril 1991.

64 Selon les termes employés par le commissaire du gouvernement dans ses conclusions sur CE, sect., 3 novembre 1997, n 153686, RFDA 1998, p. 90.

65 CAA Lyon, 30 sept. 1993, RFDA 1994, "Responsabilité hospitalière et risques thérapeutiques : de la présomption de faute à la responsabilité sans faute ", obs. Bon Pierre, p. 99 et 100 .

66 CE, ass., 9 avril 1993, n 69336, Bianchi, RFDA 1993, p. 573, concl. DAëL M. ; AJDA 1993, p. 334, chron. Mauguë Christine et Touvet Laurent, p. 126, concl. Dä̈L M. ; D. 1994, S.C. 65, obs. Bon Pierre et Terneyre Philippe ; JCP 1993, II, 22061, note Moreau Jacques; LPA 19 mai 1993, note Thiriez Frédéric; RDP 1993, p. 1099, note PAILlet Michel ; Rev. adm. 1993, p. 561, note FraisseIx Patrick. 
prévisible de cet état, et présentant un caractère d'extrême gravité ». L'hôpital fut condamné à verser à la mère 150000 francs au titre du préjudice subi et 623675 francs à la Caisse primaire d'assurance maladie au titre des frais dus à l'hospitalisation de l'enfant.

Sur pourvoi de l'hôpital, le Conseil d'État, dans son arrêt du 3 novembre $1997^{67}$, décida que " lorsqu'un acte médical nécessaire au diagnostic ou au traitement du patient présente un risque dont l'existence est connue mais dont la réalisation est exceptionnelle et dont aucune raison ne permet de penser que le patient y soit particulièrement exposé, la responsabilité du service public hospitalier est engagée si l'exécution de cet acte est la cause directe de dommages sans rapport avec l'état initial du patient comme avec l'évolution prévisible de cet état et présentant un caractère d'extrême gravité ".

Par cette décision, le Conseil d'État a étendu le champ d'application de la jurisprudence Bianchi. Surtout, l'arrêt permet de ne pas exclure certaines victimes, par la substitution du terme " patient " au terme malade, suivant en cela les conclusions du Commissaire au gouvernement.

En fait, la question centrale qui se posait à l'occasion de cette affaire était celle de savoir si "l'acte médical à l'origine du dommage était nécessaire au traitement de l'enfant ". Certes l'anesthésie évitait à l'enfant de souffrir et donc en tant qu'accessoire à l'acte chirurgical, elle pouvait être considérée comme nécessaire au traitement du malade. Empêcher la douleur de l'enfant devenait donc un motif déterminant. Cependant, et comme ont pu l'écrire certains commentateurs ${ }^{68}$, le présupposé du Commissaire du gouvernement dans l'affaire étudiée était discutable, à savoir considérer que l'enfant était dans la situation d'un malade qui devait subir un traitement nécessaire à sa guérison. Or l'enfant était en parfaite santé lors de son hospitalisation et l'opération envisagée n'avait aucun caractère thérapeutique. Les choses étaient très clairement posées. Le Conseil d'État adopte, dès lors, une conception souple de la notion d'acte médical, englobant ceux dépourvus de fin thérapeutique.

La loi $\mathrm{n}^{\circ}$ 2002-303 du 4 mars 2002 a consacré le régime de responsabilité pour faute ${ }^{69}$ et substitué à l'indemnisation fondée sur le principe de

67 CE, sect., 3 novembre 1997, n 153686, LPA 28 janvier 1998, n 12, p. 20, "L'extension de la jurisprudence Bianchi aux anesthésies générales pratiquées lors d'interventions dépourvues de fin thérapeutique ", note Allorteau Stéphane ; LPA 9 janvier 1998, n 4, p. 16, «Une nouvelle avancée du risque comme fondement de la responsabilité de l'hôpital public ", note LecocQ Pierre-André ; RFDA 1998, p. 90, "La responsabilité des services hospitaliers : extension de la responsabilité sans faute, le cas des accidents anesthésiques ", concl. du commissaire du gouvernement PÉCresse Valérie, sur CE, 3 novembre 1997.

68 Alloiteau Stéphane, note précitée.

69 C. santé publ., art. L. 1142-1-I. 
responsabilité sans faute, l'indemnisation de l'aléa thérapeutique au titre de la solidarité nationale ${ }^{70}$.

Le débat rebondit alors : les actes médicaux sans finalité thérapeutique entrent-ils dans le champ d'application de la loi de 2002 ? Comme le souligne la Commission nationale des accidents médicaux dans une recommandation du 27 mars 2009, la loi faisant état " de dommage imputable à une activité de prévention, de diagnostic ou de soins, seraient exclus en première analyse, tous les actes médicaux sans finalité thérapeutique ». Cependant, et à partir d'un examen du cas de la chirurgie esthétique, la Commission considère que les dispositions législatives ne permettent pas d'écarter de l'application de la loi l'ensemble des actes médicaux sans finalité thérapeutique, de même qu'elle ne permet pas de définir un critère tiré de l'intention du patient ou de la finalité cultuelle de l'acte en cause. De façon laconique, la Commission se contente ici de rappeler la jurisprudence du Conseil d'État de 1997. Dès lors et à titre de conclusion de sa recommandation, la Commission constate qu' " en l'état actuel des textes législatifs, comme de la jurisprudence, il n'est pas possible d'exclure du champ d'application de la loi du 4 mars 2002, les actes médicaux sans finalité thérapeutique directe, qu'il s'agisse de la chirurgie esthétique ou d'actes médicaux à finalité cultuelle ${ }^{71}$.

70 C. santé publ., art. L. 1142-1-II.

71 Dans une question écrite au gouvernement le 16 septembre 2008, une députée émettait l'idée de la création d'un contrat d'assurance circoncision proposé à la naissance des enfants mâles et destiné à prendre en charge les frais de réalisation de l'acte chirurgical. La proposition envisageait " sous certaines conditions préservant le principe de lä̈cité ", une participation limitée de l'assurance maladie. La députée invoquait que la circoncision rituelle pratiquée par un chirurgien, coûtait entre 500 et 1000 euros à la famille et soulignait que, compte tenu de cet état de fait, nombre de circoncisions rituelles étaient effectuées sous couvert d'une indication médicale car les familles ont tendance à insister auprès du médecin, voire à ne pas décalotter l'enfant afin qu'il développe un véritable phimosis impliquant la prise en charge par l'assurance maladie. La députée ajoutait que "la pratique ancestrale de la circoncision implique un taux important de complications (hémorragies, surinfections, plaies et mutilations du gland) nécessairement prises en charge par l'assurance maladie et générant un surcoût pour l'assurance maladie ". La députée citait le chiffre de 9 millions d'euros concernant cette couverture de l'acte, d'après l'évaluation d'un médecin de la CNAM et avancé en 2006, lors du congrès annuel de l'Association française des urologues. La réponse du ministre en charge de la santé, le 30 juin 2009, rappelait que le champ de l'assurance maladie tel que défini par l'article L. 321-1 du code de la sécurité sociale couvre les soins nécessaires au maintien ou au rétablissement de l'état de santé des personnes. Ainsi en est-il des actes thérapeutiques destinés à la réduction d'un phimosis. En revanche, la circoncision rituelle pour motif religieux n'entre pas dans ce cadre, de même que les actes médicaux pratiqués pour d'autres motifs personnels, esthétiques ou de confort. " Ainsi par exemple, un acte de chirurgie réparatrice sera remboursé lorsqu'il est consécutif aux lésions provoquées par le traitement d'un cancer, ce qui n'est pas le cas des interventions à visée purement esthétiques. Il s'agit d'un principe simple, aisément compréhensible et il n'est pas envisageable d'y déroger. En ce qui concerne les problèmes de santé induits par la pratique de la circoncision, il convient de rappeler que l'acte médical relève, aux termes de l'article 
Toutefois, une récente modification législative intervenue à l'hiver 2014 remet en cause cette situation. En effet, la dernière loi de financement de la sécurité sociale ${ }^{72}$ est venue restreindre considérablement le bénéfice de l'indemnisation par l'Office national d'indemnisation des accidents médicaux (ONIAM). Le législateur prévoit désormais que les actes dépourvus de finalité préventive, diagnostique ou reconstructive ainsi que ceux permettant leur préparation (prise d'antalgique ou anesthésie) et leur suivi sont exclus d'une indemnisation au titre de la solidarité nationale. Par conséquent, l'indemnisation n'est désormais plus conditionnée par la nature de l'acte médical mais par sa finalité. Dès lors, les dommages résultant d'une circoncision rituelle, acte médical à la finalité préventive discutée et à la finalité thérapeutique non reconnue, ne seront plus pris en charge par l'ONIAM en cas d'accident médical ${ }^{73}$.

Cette disposition législative bien que motivée par un souci d'économie en faveur de l'assurance maladie n'est pas exempte de critique. En premier lieu, elle prend le contrepied de la position prônée jusque récemment par la Cour de cassation ${ }^{74}$. Mais au-delà de cette résistance législative, cette disposition entraîne en second lieu une rupture d'égalité en matière de réparation, selon que l'acte médical dommageable revêt ou non une finalité thérapeutique. Enfin, elle pose de nouveau la question de la définition de ce critère de "finalité thérapeutique ». En la matière, la tentation est grande de se référer au critère retenu par la matière fiscale selon laquelle les prestations à finalité thérapeutique ${ }^{75}$ sont celles prises en charge par l'assurance maladie ${ }^{76}$. Ce critère a certes le mérite de la simplicité mais il encourage de facto la dissimulation de la circoncision rituelle en un acte médical à visée thérapeutique et ce afin de permettre son indemnisation au titre de la solidarité nationale.

L. 4161-1 du code de la santé publique, de la compétence exclusive du médecin, et partant doit être réalisé dans les mêmes conditions de sécurité et de suivi médical quelles que soient ses modalités de prise en charge. Le praticien engage sa responsabilité et s'expose à des recours. Concernant enfin la création d'une assurance sur ce sujet, l'absence d'aléa rend inapplicable le principe même de l'assurance. Il ne peut être envisagé, en tout état de cause, la participation de l'assurance maladie à un dispositif de cette nature. "

72 Loi n 2014-1554 du 22 décembre 2014 de financement de la sécurité sociale pour 2015, JO 24 déc. 2014, p. 21748.

73 Sauf à dissimuler l'acte en une pratique à visée préventive mais sous peine cette fois d'encourir une poursuite au pénal.

74 V. Cass. $1^{\text {re }}, 5$ févr. 2014, no 12-29.140, D. 2014, p. 697 : La Haute juridiction considère que les actes de chirurgie esthétique ainsi que les actes qui leur sont préparatoires sont des actes de soins, et que par conséquent les dommages non fautifs qui en résultent ouvrent donc droit à réparation au titre de la solidarité nationale.

75 Seules à pouvoir bénéficier de l'exonération de TVA. Vioujas Vincent, «L'exclusion des actes de chirurgie esthétique du dispositif de réparation des préjudices au titre de la solidarité nationale. Une réforme problématique ", D. 2014, n 44, p. 2535. 


\section{LA REMISE EN CAUSE INDIRECTE DE L'IMPUNITÉ DE LA CIRCONCISION RITUELLE}

Au regard du droit pénal, la question qui se pose tient à la qualification de l'acte de circoncision. Deux possibilités sont, ici, envisageables.

Larticle 222-9 du Code pénal selon lequel «Les violences ayant entrâné une mutilation ou une infirmité permanente sont punies de dix ans d'emprisonnement et de $150000 €$ d'amende », est-il applicable à la circoncision rituelle? En d'autres termes, la circoncision constitue-t-elle une mutilation? II n'en est rienselon les dictionnaires delalanguefrançaise, quidéfinissentla mutilation comme une atteinte (volontaire) à l'intégrité physique entraînant la perte d'un membre ou d'un organe ${ }^{77}$. La jurisprudence, certes peu fournie, s'inscrit dans cette définition. Ainsi, des femmes ayant subi une ligature des trompes, réalisée sans leur consentement au cours d'une césarienne, ne sont pas considérées comme ayant été mutilées ${ }^{78}$. Une personne ayant perdu de façon quasi complète la lèvre inférieure de la bouche a, quant à elle, subi une infirmité permanente et non une mutilation ${ }^{79}$. Mais l'arrachement du pavillon de l'oreille ${ }^{80}$ de même que récemment l'arrachement d'un bout de lobe de l'oreille ${ }^{81}$ ont pu être qualifiés de mutilation. Si le principe d'interprétation stricte qui régit le droit pénal s'oppose à ce que la perte d'un morceau de peau, qui n'est ni un membre ni un organe, soit qualifiée de mutilation, certains auteurs qualifient tout de même la circoncision de mutilation, en tant qu'elle constitue une ablation totale ou partielle du prépuce, indépendamment de l'absence de conséquences défavorables pour le sujet ${ }^{82}$. La qualification s'impose, selon Rémy Libchaber, sans hésitation particulière, «l'ablation définitive d'un élément corporel, fût-il d'une utilité dérisoire, peut difficilement recevoir une autre qualification ${ }^{83}$. Toutefois,

77 Dictionnaire Larousse. Selon le Petit Robert, la mutilation est « la perte accidentelle ou l'ablation d'un membre, d'une partie externe du corps, qui cause une atteinte irréversible à l'intégrité physique ». Au titre des mutilations sexuelles, ce dictionnaire mentionne la castration, l'excision et l'infibulation - mais pas la circoncision.

78 Cass. crim., 19 janv. 2005, Dr. pén. 2005, comm. 55, obs. Véron Michel.

79 Cass. crim., 31 oct. 2012, n $12-85458$.

80 Cass. crim., 8 mars 1912, Bull. crim. $\mathrm{n}^{\circ} 138$.

81 CA Chambéry, 6 janv. 2011, JurisData, inédit.

82 Rassat Michèle-Laure, Droit pénal spécial, Paris, Dalloz, coll. "Précis ", 7e éd., 2014, $\mathrm{n}^{\circ} 342$, p. 384.

83 Libchaber Rémy, "Circoncision, pluralisme et droits de l'homme ", D. 2012, chron., p. 2044. 
il est évident que la circoncision rituelle n'est pas comparable à l'excision ${ }^{84}$ qui répond sans aucune hésitation à la qualification de mutilation.

La seconde possibilité de qualification est celle de violence ${ }^{85}$. La circoncision serait alors passible des articles 222-11 ou 222-13 du Code pénal à condition qu'une incapacité totale de travail (ITT) soit constatée par le médecin légiste. Dans le premier cas de figure (ITT supérieure à huit jours), la peine s'élève à trois ans d'emprisonnement et 45000 euros d'amende. Cette infraction est punie de cinq ans d'emprisonnement et de 75000 euros d'amende lorsqu'elle est commise sur un mineur de quinze ans, ce qui représente une circonstance aggravante. Dans le second cas (ITT inférieure ou égale à huit jours ou absence d'ITT), la peine est de trois ans d'emprisonnement et de 45000 euros d'amende lorsque l'infraction est commise sur un mineur de quinze ans. Quant à l'élément moral de l'infraction, la personne qui pratique volontairement une circoncision ou le parent qui fait volontairement pratiquer une circoncision sur un enfant remplissent l'élément moral de l'infraction (ils agissent en connaissance de cause, c'est-à-dire en ayant conscience du geste qu'ils accomplissent).

Toutefois, la permission de la loi issue de la coutume peut-elle justifier l'accomplissement d'un rite religieux enfreignant la loi pénale? Tel est le cas lorsque la violence est légère et ne laisse pas de trace définitive. Mais les violences allant pour certaines jusqu’à la mutilation et emportant des conséquences durables et défavorables pour la victime ne peuvent pas être justifiées, comme par exemple l'excision. Concernant la circoncision rituelle, certains auteurs considèrent que la coutume peut être un fait justificatif ${ }^{86}$.

La question relative à la pénalisation de la circoncision rituelle n’a pas été soulevée en jurisprudence, excepté, mais peu clairement, dans un arrêt de la Cour d'appel de Grenoble en date du 7 novembre $2012^{87}$. La mère avait fait procéder à la circoncision de son fils par un infirmier. Dans cette affaire, le médecin avait fixé une ITT de 20 à 30 jours après avoir constaté " un traumatisme psychologique secondaire et un syndrome douloureux mal géré par la personne ayant pratiqué la circoncision ». Condamnée par le tribunal

84 Sur l'excision, voir notamment, ForTier Vincente, "L'excision, quelle réponse pénale ? ", Arch. Pol. crim., n 21, 1999, p. 41-54; du même auteur, "Circoncision, excision : des atteintes à l'intégrité du corps humain », in Fortier Vincente et Vialla François (dir.), $L a$ religion dans les établissements de santé, Bordeaux, Les Études hospitalières, coll. "Regards croisés ", 2013, p. 235-264.

85 Francis CABALLERo considère que la circoncision pourrait être qualifiée de crime de violences aggravées avec armes sur des mineurs ayant entraîné une amputation (Droit du sexe, Paris, LGDJ-Lextenso éditions, 2010).

86 Desportes Frédéric, Le Gunehec Francis, Droit pénal général, Paris, Economica, 15 éd., 2008. Contra : Choain Christine, note sous Cass. $1^{\text {re }}, 26$ janv. 1994, D. 1995, jurispr., p. 226.

87 CA Grenoble, 7 novembre 2012, JurisData n 2012-034508. 
correctionnel, la mère est relaxée des faits de violences volontaires en cour d'appel : l'élément moral de l'infraction faisait défaut car, en raison de son isolement, la mère n'avait pas eu conscience de contribuer à un acte de violence volontaire sur l'enfant. Arrivée depuis peu de temps en France, elle s'était renseignée auprès de la communauté maghrébine et pensait que l'intervenant était qualifié pour procéder à la circoncision. Selon la cour, «il n’apparait pas qu'elle ait eu conscience de contribuer à un acte de violences volontaires sur son fils ». Est-ce à dire qu'une personne " non isolée ", autrement dit bien intégrée qui fait circoncire son enfant dans de telles circonstances (par un non-médecin) commettrait volontairement un acte de violence?

Si l'on considère que la circoncision peut être qualifiée de mutilation, il convient alors de tenir compte de la loi $\mathrm{n}^{\circ} 2013-711$ du 5 août 2013 portant diverses dispositions d'adaptation dans le domaine de la justice en application du droit de l'Union Européenne et des engagements internationaux de la France. Cette loi crée un contexte nouveau en édictant une nouvelle infraction en matière de mutilations sexuelles ${ }^{88}$. Le nouvel article 227-24-1 du Code pénal issu de la loi du 5 août 2013 dispose en effet : "Le fait de faire à un mineur des offres ou des promesses ou de lui proposer des dons, présents ou avantages quelconques, ou d'user contre lui de pressions ou de contraintes de toute nature, afin qu'il se soumette à une mutilation sexuelle est puni, lorsque cette mutilation n'a pas été réalisée, de cinq ans d'emprisonnement et de 75000 euros d'amende.

Est puni des mêmes peines le fait de provoquer directement autrui à faire subir une mutilation sexuelle à un mineur, lorsque cette mutilation n'a pas été réalisée, ou d'en faire l'apologie. "

Il s'agissait, par la loi du 5 août 2013, de transposer des dispositions de la convention du Conseil de l'Europe sur la prévention et la lutte contre la violence à l'égard des femmes et la violence domestique, signée à Istanbul, le 11 mai 2011. Cette convention a pour objet de prévoir des règles minimales en matière de prévention, de prise en charge des victimes, ainsi que de poursuite et de répression des auteurs d'infractions auxquelles les femmes sont particulièrement exposées, telles que les violences sexuelles (viols et agressions sexuelles), les violences physiques et psychologiques, le harcèlement, les mariages forcés, les mutilations génitales ou encore les "crimes d'honneur".

Larticle 16 de la loi du 5 août 2013 opère trois modifications au sein du Code pénal destinées à mettre la législation française en conformité avec trois stipulations de la convention, notamment en incriminant l'incitation non suivie d'effet à subir une mutilation sexuelle.

88 Pour un commentaire, Benillouche Mikaël, "L'interdiction des mutilations sexuelles : entre confirmation et révolution... ", RDLF, chron. $n^{\circ}$ 6, 2014 (www.revuedlf.com). 
L'article 38 de la convention d'Istanbul du 11 mai 2011 prévoit l'obligation pour les États de sanctionner pénalement les mutilations génitales :

«Les Parties prennent les mesures législatives ou autres nécessaires pour ériger en infractions pénales, lorsqu'ils sont commis intentionnellement :

« a) l'excision, l'infibulation ou toute autre mutilation de la totalité ou partie des labia majora, labia minora ou clitoris d'une femme ;

«b) le fait de contraindre une femme à subir tout acte énuméré au point $a$ ou de lui fournir les moyens à cette fin;

« C) le fait d'inciter ou de contraindre une fille à subir tout acte énuméré au point a ou de lui fournir les moyens à cette fin. "

Le droit pénal français est déjà conforme aux exigences des paragraphes $a$ et $b$ de cet article. En revanche, s'agissant du $c$, si la contrainte qu'il mentionne est bien incriminée en tant que violences lorsqu'elle est suivie d'effet, tel n'est pas le cas pour l'incitation d'une jeune fille à subir une mutilation génitale lorsque cette incitation n'est pas suivie d'effet.

Ce nouveau délit est donc introduit dans un nouvel article 227-24-1 du Code pénal qui s'insère au sein de la section relative à la mise en péril des mineurs du chapitre consacré aux atteintes aux mineurs et à la famille ${ }^{89}$.

Il faut ici souligner que le projet de loi n'a pas limité l'application de cette disposition nouvelle aux seules jeunes filles, mais l'a rendue applicable à tout mineur, fille ou garçon. Le rapport 840 indique en effet que "Selon les informations communiquées par le ministère de la Justice, cette application à tous les mineurs indépendamment de leur sexe a été considérée comme nécessaire pour

89 Selon le rapport 840, l'élément matériel de l'infraction pourra être caractérisé par plusieurs comportements : il pourra s'agir d'offres, de promesses, de propositions de dons, présents ou avantages quelconques, de pressions ou de contraintes de toute nature.

Le nouvel article 227-24-1 requiert, en plus de l'intention de commettre l'infraction, un dol spécial constitué par l'intention d'amener le mineur à se soumettre à une mutilation sexuelle. Cette incitation ne devra pas avoir été suivie d'effet : dans ce cas, l'infraction de violences ayant entraîné une mutilation sera constituée, la personne ayant incité la victime à s'y soumettre étant alors complice de cette infraction de violences.

La victime de l'infraction devra être mineure au moment des faits, conformément au texte de la convention : en effet, le c utilise le terme " fille", alors que les a et b utilisent le terme "femme». Le rapport explicatif sur la convention met en évidence le fait que l'objectif du c est d'apporter aux mineurs une protection particulière allant plus loin que celle accordée aux femmes adultes : "L'alinéa c érige en infraction pénale le fait d'apporter une assistance à l'auteur des faits visés à l'alinéa a en incitant ou contraignant une fille à subir l'excision, l'infibulation ou toute autre mutilation de ses labia majora, labia minora ou clitoris, ou en lui fournissant le moyen à cette fin. Cette partie de la disposition s'applique uniquement aux filles, et inclut les situations dans lesquelles toute personne, et en particulier les parents, les grands-parents ou autre parent contraignent leur fille ou parent à subir ce procédé. Les rédacteurs ont estimé qu'il était important de distinguer entre adultes victimes et enfants victimes, car ils ne souhaitaient pas ériger en infraction pénale le fait d'inciter des femmes adultes à effectuer un des actes listés à l'alinéa a ». 
ne pas introduire de rupture d'égalité entre les mineurs. Certes, les mutilations génitales féminines sont plus fréquentes en raison de la persistance de traditions observées par certaines communautés, comme le note le rapport explicatif sur la convention. Pour autant, de la même façon que les dispositions actuelles sur les mutilations peuvent s'appliquer à un homme qui aurait subi une mutilation génitale, le ministère de la Justice a fait valoir qu'il ne serait pas légitime d'exclure les jeunes garçons du champ d'application du nouveau délit d'incitation non suivie d'effet à subir une mutilation. Dans la mesure où la rédaction proposée permet bien d'incriminer l'incitation non suivie d'effet à subir une mutilation génitale adressée à une jeune fille, elle paraît conforme à la convention et ce, bien qu'elle ait un champ d'application plus large permettant également de protéger les jeunes garçons".

Ce nouveau délit sera puni de cinq ans d'emprisonnement et 75000 euros d'amende. Il pourra également être sanctionné des peines complémentaires prévues aux articles 227-29 et 227-31.

Le législateur n'a-t-il pas, par là-même, ouvert la voie à de possibles poursuites en cas de certains faits liés à la circoncision?

\section{Conclusion}

Aux termes de cette étude de la situation française à l'égard de la circoncision rituelle, il ressort très clairement qu'il n'est pas possible de conclure à une licéité de principe de l'acte, même s'il est médicalisé. Car, à tout le moins, la condition tenant au consentement du sujet fait défaut. Le droit de la famille, certes, encadre la pratique. En érigeant la circoncision rituelle en acte non usuel de l'autorité parentale, il la soumet à la co-décision des parents. Bien que les juges sanctionnent son accomplissement dans des conditions non respectueuses des droits de l'autre parent, la circoncision rituelle demeure une atteinte à l'intégrité physique du mineur. Enfin, si le droit pénal, jusqu'ici, n’a pas été mobilisé, toutefois ses nouvelles dispositions par la généralité de ses termes et non point leur imprécision, chacun s'accordant, en effet, sur le sens de l'expression « mutilation sexuelle ", accroît encore la fragilité de la situation de la circoncision rituelle.

Finalement et quel que soit l'angle juridique d'approche de la circoncision rituelle, le questionnement demeure irrésolu et la quête de certitude du juriste insatisfaite : faut-il conforter la pratique du rite au nom de la liberté des religions au détriment peut-être de celle de l'enfant? Quel intérêt (de l'enfant, des parents, des religions) faut-il faire prévaloir ? L'impératif de sécurité juridique ne doit-il pas conduire à prendre une position dénuée d'ambiguïté ? Mais dans un tel cas de figure, des effets pervers ne doivent-ils pas être redoutés? 